\title{
Angular Properties of Delaunay Diagrams in Any Dimension
}

\author{
D. Schmitt and J.-C. Spehner \\ Laboratoire de Modélisation et Algorithmique Géométrique, \\ Faculté des Sciences et Techniques, Université de Haute-Alsace, \\ 4 rue des Frères Lumière, 68093 Mulhouse Cédex, France \\ \{D.Schmitt, JC.Spehner\}@univ-mulhouse.fr
}

\begin{abstract}
Equiangularity (also called max-min angle criterion) is a well-known property of some planar triangulations that refine the Delaunay diagram. In this paper we generalize the notion of equiangularity to decompositions in inscribable polygons and we show that it characterizes the planar Delaunay diagram, even if more than three sites are cocircular. This result does not extend to higher dimensions. However, we characterize the Delaunay diagram in any dimension by a kind of dual property that we prove both with line angles and with solid angles. We also establish a local equiangularity of Delaunay diagrams in any dimension, and an angular characterization of self-centered diagrams. Finally, we show that these angular properties can, when appropriately defined, be generalized to the farthest point Delaunay diagram.
\end{abstract}

\section{Introduction}

Given a set $S$ of points in the plane called sites, the problem of triangulating $S$, that is, decomposing the convex hull of $S$ in disjoint triangles whose vertices are the sites of $S$, has been studied extensively. Among these triangulations, Delaunay triangulations (whose triangles circumcircles contain no site in their interior) seem to be the most regular [2], [5], [9], [10]. Indeed, a Delaunay triangulation maximizes the minimum angle of its triangles, and moreover maximizes lexicographically the increasing sequence of these angles [5]. Such a triangulation is said to be globally equiangular. The Delaunay triangulation definition extends easily to higher dimensions, but very few results are known so far. Some metric properties of planar Delaunay triangulations have been generalized in any dimension [11], [12]. If no $d+2$ sites of $S$ are cospherical, the Delaunay triangulation of $S$ is dual to the Voronoi diagram of $S$. Otherwise in degenerate cases, the dual of the Voronoi diagram may not be a triangulation but a decomposition of the convex hull of $S$ in disjoint convex inscribable polytopes whose vertices are the sites of $S$ (see Fig. 1). 


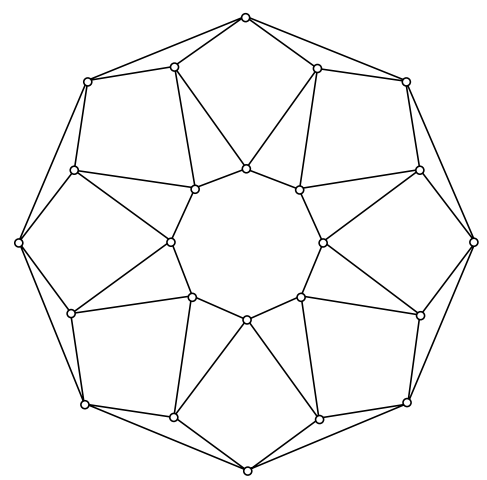

Fig. 1. A "degenerate" Delaunay diagram.

Such a decomposition is called an inscribable diagram of $S$ and the unique dual of the Voronoi diagram is the Delaunay diagram denoted by $\operatorname{Del}(S)$. The circumsphere of each Delaunay region contains no site in its interior and passes only through the sites that are vertices of this region. Thus, Delaunay triangulations are refinements of the Delaunay diagram.

In Section 2 we characterize the Delaunay diagram in any dimension through a property of its facets. All other results will be consequences of this one.

In Section 3 we introduce another definition of angles which generalize those used to establish equiangularity of planar Delaunay triangulations, and we extend this property to planar Delaunay diagrams.

Although these angles can be generalized in different ways in higher dimensions, we show in Section 4 that none extend equiangularity to higher-dimensional Delaunay diagrams. However, by adding up these angles facet per facet, we define in Section 5 a property which is - in a certain way-dual to equiangularity. We also prove that this property characterizes Delaunay diagrams in any dimension.

These new angles also allow us to characterize self-centered inscribable diagrams (for which every region contains its circumcenter), and to establish a kind of local equiangularity of Delaunay diagrams in any dimension.

In Section 8 we show that Delaunay diagrams can also be characterized in dimension greater than two with solid angles.

The order- $k$ Delaunay diagram of a $d$-dimensional set $S$ of $n$ sites is the orthogonal dual of the order- $k$ Voronoi diagram of $S$ such that every order- $k$ Delaunay vertex is the center of gravity of the $k$ sites defining its dual order- $k$ Voronoi region [1], [3], [14], [13]. By using a homothety centered at the center of gravity of $S$ and with factor $-1 /(n-1)$, the order- $(n-1)$ Delaunay diagram of $S$ can be mapped into an inscribable diagram whose vertices are those of the convex hull of $S$. This inscribable diagram is called the farthest point Delaunay diagram of $S$, and is denoted by $\operatorname{FDD}(S)$. The interior of the circumsphere of every region $p$ of $\operatorname{FDD}(S)$ contains all the sites of $S \backslash \delta(p)(\delta(p)$ denotes the boundary of $p$ ).

Eppstein [7] has proved that the triangulation of the vertices of a convex hull of a planar point set that minimizes the increasing angles sequence, is a farthest point 
Delaunay triangulation. In Section 9 we show that angular properties of the farthest point Delaunay diagram are opposite to properties of $\operatorname{Del}(S)$.

\section{Illegal Facets in Inscribable Diagrams}

Given a set $S$ of sites in a $d$-dimensional space, Delaunay [4] has shown that Delaunay triangulations of $S$ are the only triangulations of $S$ whose every facet $f$ common to two tetrahedrons $p$ and $q$ is such that the interior of the circumsphere of $p$ contains no vertex of $q$. Such a facet $f$ is said to be locally optimal [8] or not illegal [5].

However, these facets do not characterize Delaunay diagrams if more than $d+1$ sites are cospherical. Indeed, the diagonal of a square for example is locally optimal, but is not a Delaunay edge: the circumsphere of a Delaunay region $p$ passes only through the sites that are vertices of $p$. Therefore we modify the previous definitions to take degeneracies into account.

Recall that a diagram in a $d$-dimensional euclidean space $E$ is a partition of $E$ in a finite number of 0 -faces (called vertices), of 1-faces (called edges), of 2-faces, ..., of $(d-1)$-faces (called facets), and of $d$-faces (called regions). For a set $S$ of sites in $E$, let $\operatorname{Dins}(S)$ be the set of inscribable diagrams whose set of vertices is $S$. Faces (other than 0 -faces) of these diagrams are open convex inscribable polytopes, and the complement of the convex hull of $S$ is the only unbounded face. $\operatorname{Dins}(S)$ contains, for instance, the Delaunay diagram and all triangulations of $S$.

A facet $f$ of an inscribable diagram is said to be illegal if the two regions $p$ and $q$ having $f$ as a common facet are bounded, and if $p$ is included in the open ball $\omega(q)$ circumscribed to $q$, that is, every vertex of $p$ belongs to the closed ball $\overline{\omega(q)}$ (in an early version [15], such a facet has been called recessive). Moreover, $p \subset \omega(q)$ is equivalent to $q \subset \omega(p)$.

Theorem 1. The Delaunay diagram $\operatorname{Del}(S)$ of a set $S$ of sites in a d-dimensional euclidean space, is the only diagram of $\operatorname{Dins}(S)$ without illegal facets.

Proof. The proof given by Delaunay [4] can be easily generalized to deal with inscribable diagrams. The proof of Theorem 7 in Section 9 gives another way to establish this result.

\section{Equiangularity of Planar Delaunay Diagrams}

In order to establish global equiangularity in planar triangulations, Edelsbrunner [5] has shown that a triangulation which maximizes lexicographically the increasing sequence of angles of its triangles is a Delaunay triangulation. However, if there are more than three cocircular sites, some Delaunay triangulations may not be globally equiangular, and there may be more than one globally equiangular triangulation. Moreover, if we consider the increasing sequence of interior angles at the vertices of the regions of an inscribable diagram, global equiangularity does not characterize Delaunay diagrams. For example, in Fig. 2 the diagram $D$ whose regions are 1234 and 145 is an inscribable 


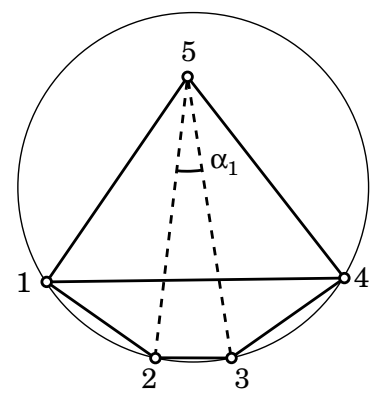

Fig. 2. A nonglobally equiangular Delaunay diagram.

diagram of the set $S=\{1,2,3,4,5\}$ but is not Delaunay. The regions of $\operatorname{Del}(S)$ are 125 , 235 , and 345. When site 3 tends toward site 2, angle $\alpha_{1}$ at vertex 5 of the region 235 tends toward zero whereas the angles of the region 1234 tend toward nonzero values. Thus, $\alpha_{1}$ may be smaller than every angle of $D$ and $\operatorname{Del}(S)$ is not globally equiangular.

Recall that the interior angle $\alpha$ at vertex $r$ of a triangle $r s t$ is equal to the half-angle under which edge $s t$ is seen from the center $O$ of the circumcircle $C$ of $r s t$ (see Fig. 3). Thus equiangularity of Delaunay triangulations can be established by using these latter angles.

Moreover, if $r^{\prime}$ is a point in the half-space limited by $s t$ and containing $r$, the nonoriented angle $\left(r^{\prime} s, r^{\prime} t\right)$ between the half-lines $r^{\prime} s$ and $r^{\prime} t$ is greater (resp. smaller) than $\alpha$ if $r^{\prime}$ is inside (resp. outside) the circle $C$.

The angles above can also be used to characterize inscribable diagrams as follows. Let $D$ be a diagram of $\operatorname{Dins}(S)$ in the plane, let $p$ be a bounded region of $D$, and let $s t$ be an edge of $p$. Let $O$ be the center of $\omega(p)$ and let $O u$ be the half-line radiating from $O$, orthogonal to $s t$ and directed to the half-plane delimited by $s t$ that does not contain $p$ (see Fig. 4).

$\mathrm{Ou}$ is called the external normal to the edge st with respect to the region $p$. The

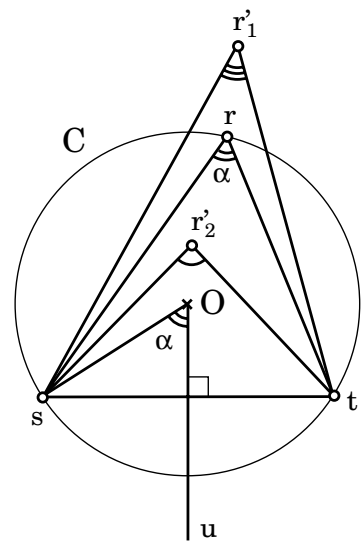

Fig. 3. For any sites $r_{1}^{\prime}, r$, and $r_{2}^{\prime}$ that are respectively outside, on, and in $C$, and on the same side of st, $\left(r_{1}^{\prime} s, r_{1}^{\prime} t\right)<\alpha=(O s, O u)=(r s, r t)<\left(r_{2}^{\prime} s, r_{2}^{\prime} t\right)$. 

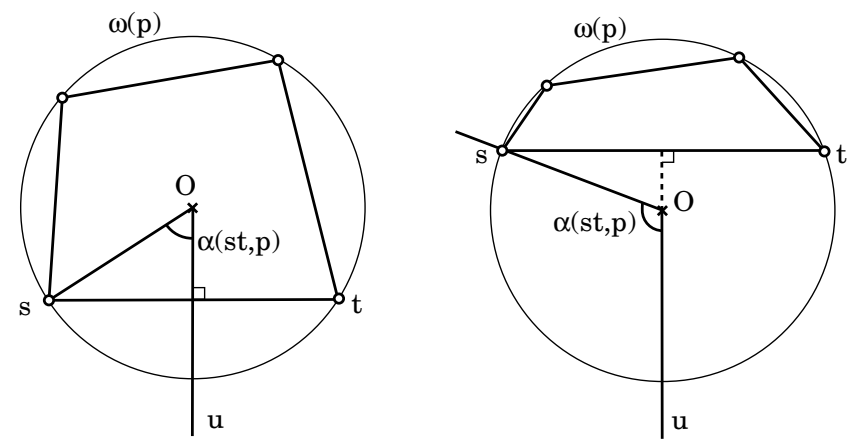

Fig. 4. Definition of the associated angle $\alpha(s t, p)$.

angle $(\mathrm{Os}, \mathrm{Ou})$ is said to be associated to the edge st with respect to the region $p$ and is denoted by $\alpha(s t, p)$. In the particular case where the region $p$ is unbounded (i.e., $p$ is the complement of the convex hull $\operatorname{conv}(S))$, we pose $\alpha(s t, p)=0$. Indeed, the straight line $s t$ is the limit of a circle passing through $s$ and $t$, and whose interior contains no site, that is, a circle whose center tends toward $-\infty$ on the bisector of $\{s, t\}$ in the half-plane limited by $s t$ and that does not contain $S$.

Unlike planar triangulations, where the number of triangles, and thus of angles, is fixed once $S$ is given, in a diagram of $\operatorname{Dins}(S)$ the length of the angles sequence depends on the diagram. So we modify the definition of equiangularity to take this fact into account. Let $D$ be a diagram of $\operatorname{Dins}(S)$ and let $\mathcal{A}(D)=\left(\alpha_{1}(D), \alpha_{2}(D), \ldots, \alpha_{m}(D)\right)$ be the increasing sequence of the nonzero angles associated to all the edges of $D$, and let $m=|\mathcal{A}(D)|$ be the length of the sequence $\mathcal{A}(D)$.

Given two diagrams $D$ and $D^{\prime}$ of $\operatorname{Dins}(S)$, we define the lexicographical order relation $<$ such that $\mathcal{A}\left(D^{\prime}\right)<\mathcal{A}(D)$ if one of these two conditions applies:

— there exists $j$ such that, $\forall i<j, \alpha_{i}\left(D^{\prime}\right)=\alpha_{i}(D)$ and $\alpha_{j}\left(D^{\prime}\right)<\alpha_{j}(D)$.

- $\mathcal{A}(D)$ is an initial subsequence of $\mathcal{A}\left(D^{\prime}\right)$ (i.e., $|\mathcal{A}(D)|<\left|\mathcal{A}\left(D^{\prime}\right)\right|$ and, $\forall i \in$ $\left.\{1, \ldots,|\mathcal{A}(D)|\}, \alpha_{i}(D)=\alpha_{i}\left(D^{\prime}\right)\right)$.

A diagram $D$ of $\operatorname{Dins}(S)$ is said to be equiangular if, $\forall D^{\prime} \in \operatorname{Dins}(S), \mathcal{A}\left(D^{\prime}\right) \leq \mathcal{A}(D)$.

It results from the previous definition that if $\mathcal{A}(D)$ is a subsequence of $\mathcal{A}\left(D^{\prime}\right)$ that is, $\mathcal{A}(D)$ is obtained by removing some elements from $\mathcal{A}\left(D^{\prime}\right)$, then $\mathcal{A}\left(D^{\prime}\right)<\mathcal{A}(D)$. Indeed, either $\mathcal{A}(D)$ is an initial subsequence of $\mathcal{A}\left(D^{\prime}\right)$, or there exist $j$ and $k>j$ such that, $\forall i<j, \alpha_{i}(D)=\alpha_{i}\left(D^{\prime}\right)$ and $\alpha_{j}(D)=\alpha_{k}\left(D^{\prime}\right)>\alpha_{j}\left(D^{\prime}\right)$.

Theorem 2. The Delaunay diagram $\operatorname{Del}(S)$ of a set $S$ of coplanar sites is the only equiangular diagram of $\operatorname{Dins}(S)$.

Proof. Let $D$ be a diagram of $\operatorname{Dins}(S)$ that admits at least one illegal edge $c$. Let $p$ and $q$ be the two regions of $D$ sharing $c$. Let $L=\delta(p) \cap S, R=\delta(q) \cap S,\{s, t\}=$ $\delta(c) \cap S=L \cap R$, and let $H$ be the restriction of $D$ to $L \cup R$.

If the sites of $L \cup R$ are cocircular, the edges of $\operatorname{Del}(L \cup R)$ are the edges of the 


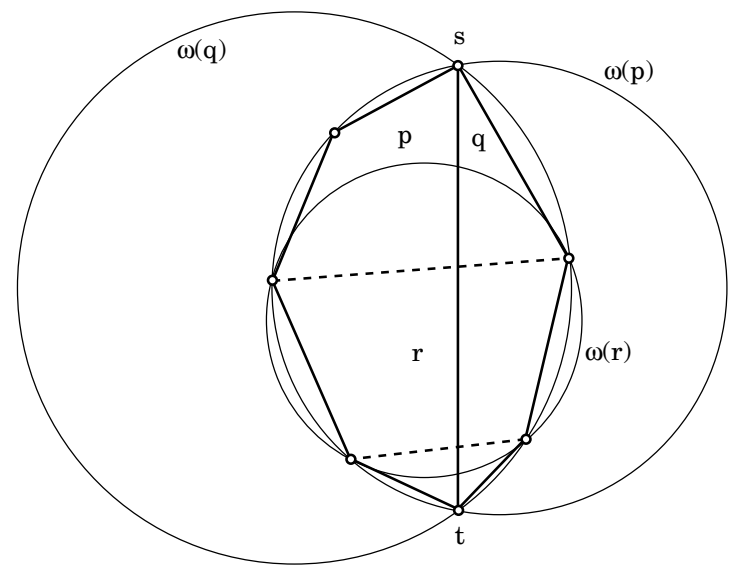

Fig. 5. Illustration for the proof of Theorem 2: the vertices of $p$ and $q$ are not cocircular.

convex polygon whose vertices are the sites of $L \cup R$, and $c$ is not an edge of $\operatorname{Del}(L \cup R)$. Therefore, $\mathcal{A}(\operatorname{Del}(L \cup R))$ is a subsequence of $\mathcal{A}(H)$, and $\mathcal{A}(H)<\mathcal{A}(\operatorname{Del}(L \cup R))$.

If the sites of $L \cup R$ are not cocircular, the sites of $L \backslash\{s, t\}$ and $R \backslash\{s, t\}$ belong respectively to $\omega(q)$ and to $\omega(p)$ (see Fig. 5). Let $r$ and $a a^{\prime}$ be respectively a region and an edge of $\operatorname{Del}(L \cup R)$ such that $\alpha\left(a a^{\prime}, r\right)=\alpha_{1}(\operatorname{Del}(L \cup R))$ is the smallest angle of $\mathcal{A}(\operatorname{Del}(L \cup R))$.

Let $O_{p}$ and $O_{r}$ be the respective centers of $\omega(p)$ and $\omega(r)$ and let $O_{r} u$ be the external normal to $a a^{\prime}$ with respect to $r$.

(1) If $\left\{a, a^{\prime}\right\} \subset L$, then $a a^{\prime}$ is an edge of $p$ and the half-line $O_{p} u$ is the external normal to $a a^{\prime}$ with respect to $p$ (see Fig. 6). Since every other vertex of $r$ belongs to $R \backslash\{s, t\}$, the circular arc of $\delta(\omega(r))$ limited by $a a^{\prime}$ and containing $r$ is included in $\omega(p)$. Moreover, the center $O_{r}$ of $\omega(r)$ belongs to the open half-line $O_{p} u$. It

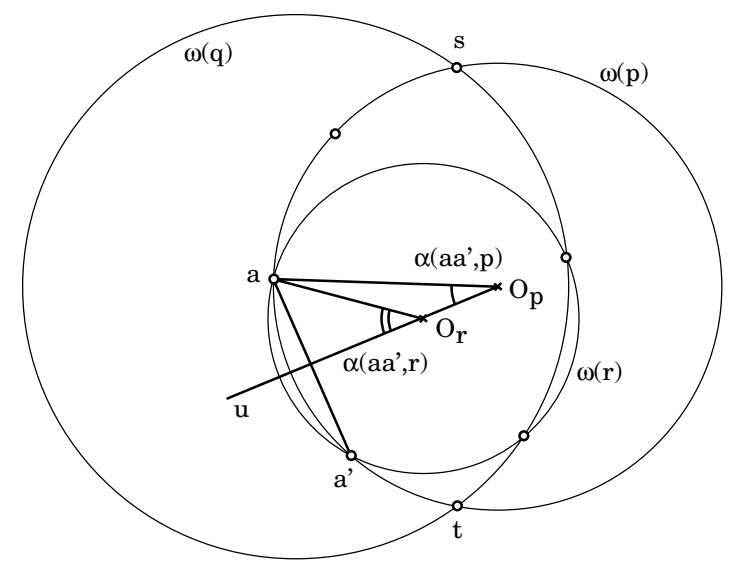

Fig. 6. Illustration for the proof of Theorem 2: part (1). 


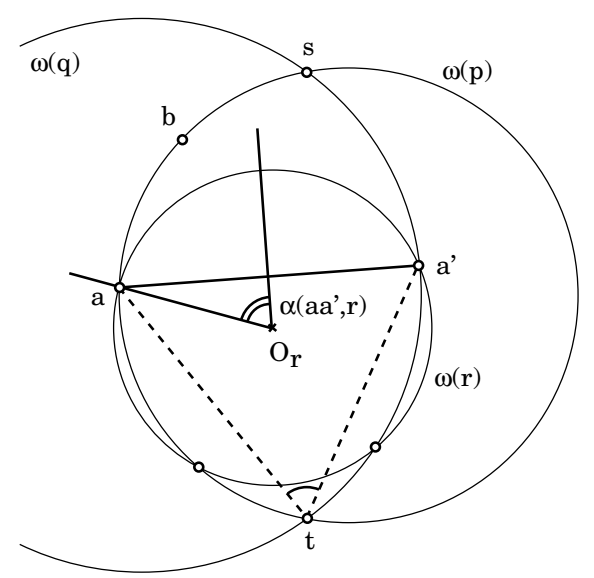

(a)

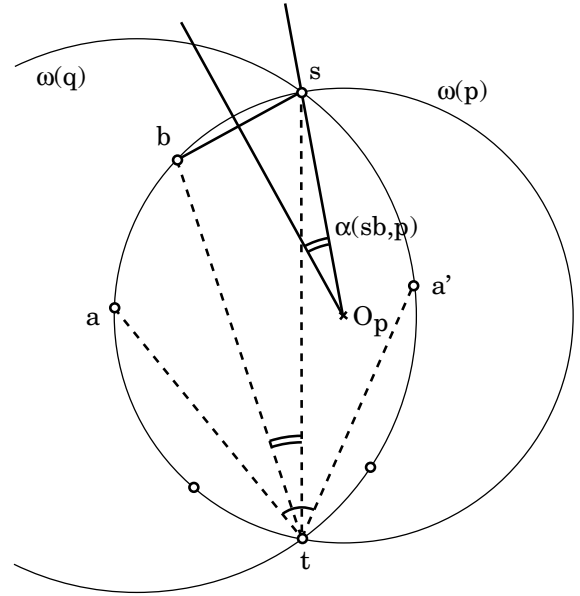

(b)

Fig. 7. Illustration for the proof of Theorem 2: part (2).

follows that $\alpha\left(a a^{\prime}, p\right)=\left(O_{p} a, O_{p} u\right)<\left(O_{r} a, O_{r} u\right)=\alpha\left(a a^{\prime}, r\right)=\alpha_{1}(\operatorname{Del}(L \cup$ $R)$ ), and since $p$ is a region of $H, \alpha_{1}(H) \leq \alpha\left(a a^{\prime}, p\right)<\alpha_{1}(\operatorname{Del}(L \cup R))$. Hence $\mathcal{A}(H)<\mathcal{A}(\operatorname{Del}(L \cup R))$.

(2) If $a \in L \backslash\{s, t\}$ and $a^{\prime} \in R \backslash\{s, t\}$, then we can suppose (within a permutation of $s$ and $t$ ) that $t$ and $r$ are on the same side of the straight line $a a^{\prime}$ (see Fig. 7(a)). Since $r$ is a region of $\operatorname{Del}(L \cup R), t$ does not belong to $\overline{\omega(r)}$ and thus $\left(t a, t a^{\prime}\right)<\alpha\left(a a^{\prime}, r\right)$. If $b$ is the neighbor of $s$ on $\delta(p)$ distinct from $t$ ( $b$ may be equal to $a$ ), then $(t b, t s)<\left(t a, t a^{\prime}\right)$ (see Fig. 7(b)). Since $t$ belongs to $\delta(\omega(p)), \alpha(s b, p)=$ $(t b, t s)<\left(t a, t a^{\prime}\right)<\alpha\left(a a^{\prime}, r\right)$. Hence $\alpha_{1}(H) \leq \alpha(s b, p)<\alpha\left(a a^{\prime}, r\right)=$ $\alpha_{1}(\operatorname{Del}(L \cup R))$ and $\mathcal{A}(H)<\mathcal{A}(\operatorname{Del}(L \cup R))$.

(3) The case $\left\{a, a^{\prime}\right\} \subset R$, and the case $a^{\prime} \in L \backslash\{s, t\}$ and $a \in R \backslash\{s, t\}$ are dealt with in the same way.

Thus, in all cases, if we replace $H$ by $\operatorname{Del}(L \cup R)$ in $D$, we obtain a diagram $D^{\prime}$ such that $\mathcal{A}(D)<\mathcal{A}\left(D^{\prime}\right)$.

This shows that the equiangular diagram (i.e., which maximizes the sequence $\mathcal{A}$ ) has no illegal edge, and Theorem 1 shows that the Delaunay diagram is the only inscribable diagram with this property.

This result holds even if we add in the sequence $\mathcal{A}$ the zero angles associated to the edges of the unbounded region: the number of these angles is the same for every inscribable diagram of a given planar point set.

\section{Equiangularity in Higher Dimensions}

In order to study angular properties in higher dimensions, we first have to define the angles we need to use. 

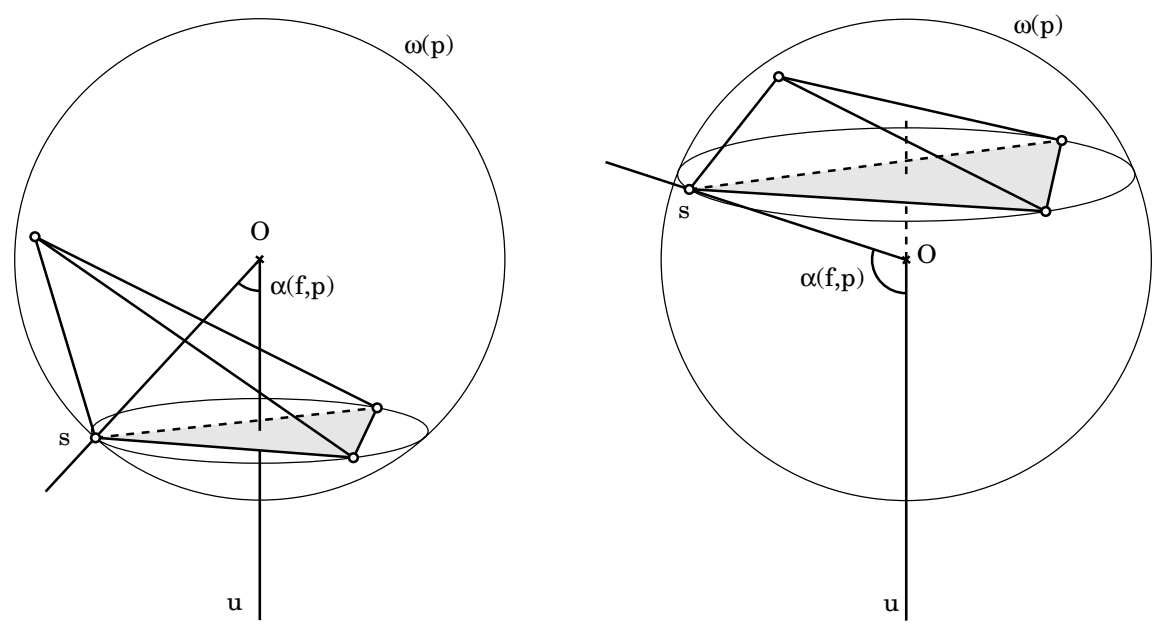

Fig. 8. In both examples, the grey facet $f$ is adjacent to a tetrahedron $p$. In the left figure, $p$ and $O$ are on the same side of facet $f$ and $0<\alpha(f, p)<\pi / 2$, whereas in the right figure, $p$ and $O$ are on both sides of $f$ and $\pi / 2<\alpha(f, p)<\pi$.

Let $p$ be a bounded region of a diagram $D$ of $\operatorname{Dins}(S)$ in the $d$-dimensional space and let $f$ be a facet of $p$. Let $O$ be the center of $\omega(p)$, let $s$ be a vertex of $f$, and let $O u$ be the half-line radiating from $O$, orthogonal to $f$ and directed to the half-space delimited by the hyperplane of $f$ that does not contain $p$ (see Fig. 8 for $d=3$ ). Note that the straight line containing $O u$ intersects $f$ in its circumcenter.

$O u$ is called the external normal to $f$ with respect to $p$, and the angle $(O s, O u)$ is said to be associated to the facet $f$ with respect to the region $p$ and is denoted by $\alpha(f, p)$. As in the plane, $\alpha(f, p)=0$ if $p$ is unbounded.

The notion of equiangularity extends easily in any dimension with these angles but, unfortunately, it does not characterize Delaunay diagrams in dimensions greater than two, as is shown in the following example (see Fig. 9).

Let $S=\{1,2,3,4,5\}$ be a three-dimensional point set in which the plane of the
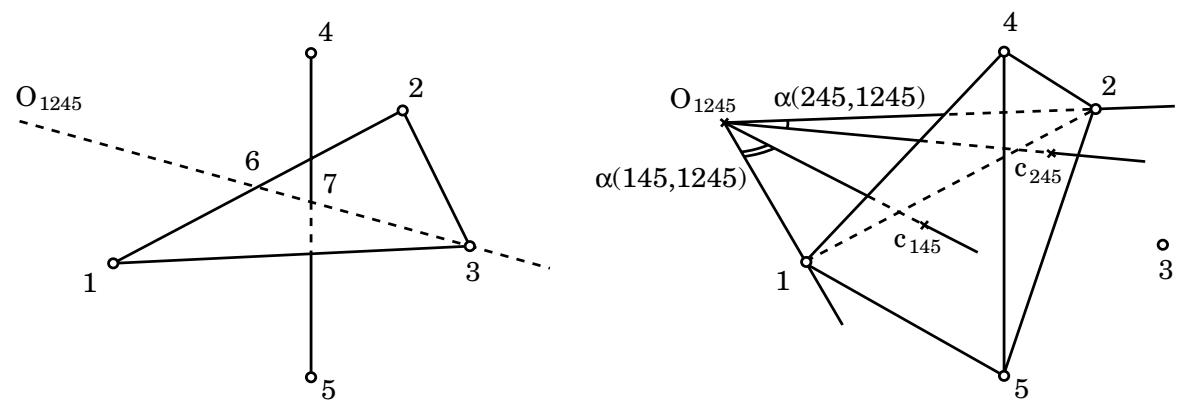

Fig. 9. The Delaunay diagram of this three-dimensional point set is not equiangular. 
equiangular triangle 123 is the bisector of $\{4,5\}$ and the straight line segment 45 cuts this plane at point 7 of the segment 36 (6 is the middle of the segment 12).

When sites 4 and 5 are near enough to the plane 123, the circumsphere of 1234 contains site 5 in its interior, and the diagram $D$ whose regions are the tetrahedrons 1234 and 1235 is the only non-Delaunay diagram of $\operatorname{Dins}(S)$. The regions of $\operatorname{Del}(S)$ are the tetrahedrons 1345,2345 , and 1245 . When point 7 tends toward point 6 , the circumcenter $O_{1245}$ of 1245 tends toward infinity whereas the circumcenters of the regions of $D$ tend toward positions at finite distances, and thus the angles associated to the facets 145 and 245 with respect to the region 1245 of $\operatorname{Del}(S)$ tend toward zero whereas the angles associated to the facets of $D$ tend toward nonzero values. Therefore, there exists a position of point 7 on the segment 36 for which the sharpest angles associated to the facets of $\operatorname{Del}(S)$ are smaller than the angles associated to the facets of $D$, and $\operatorname{Del}(S)$ is not equiangular.

Angles used in the plane can be generalized to dimension $d$ by considering other kinds of angles, such as angles associated to the $h$-faces of the diagrams (for $h \in\{1, \ldots, d-2\}$ ). However, it is straightforward to show that none characterize Delaunay diagrams.

\section{Coassociated Angles in Any Dimension}

In the previous section we have associated two angles $\alpha(f, p)$ and $\alpha(f, q)$ to each facet $f$ of an inscribable diagram with respect to the two regions $p$ and $q$ sharing $f$. The angle $\beta(f)=\alpha(f, p)+\alpha(f, q)$ is said to be coassociated to the facet $f$ (see Fig. 10).

This angle is a function of the oriented angle $\left(s O_{p}, s O_{q}\right)$ where $O_{p}$ and $O_{q}$ are the respective centers of the balls $\omega(p)$ and $\omega(q)$. $\beta(f)$ measures the way in which the two balls interpenetrate.

Theorem 3. For every finite set $S$ of sites in a d-dimensional space, the Delaunay diagram $\operatorname{Del}(S)$ is the only diagram of $\operatorname{Dins}(S)$ whose angles coassociated to the facets are all strictly smaller than $\pi$.

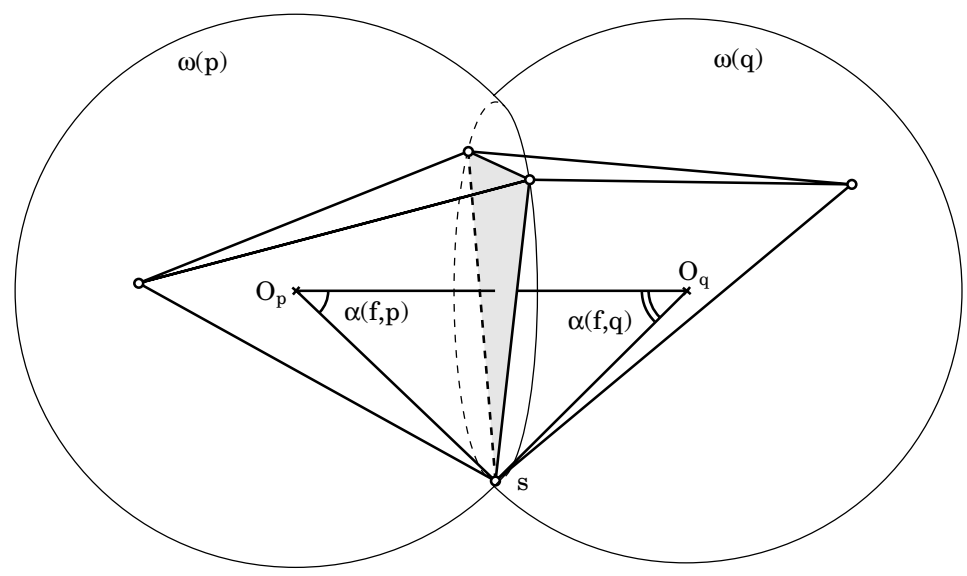

Fig. 10. Definition of coassociated angles in three dimensions. 


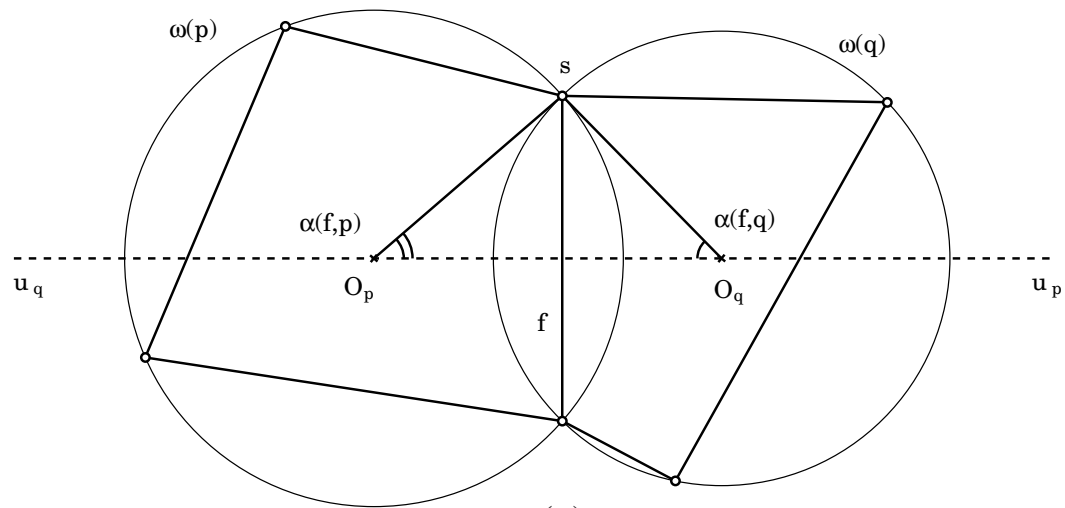

(a)

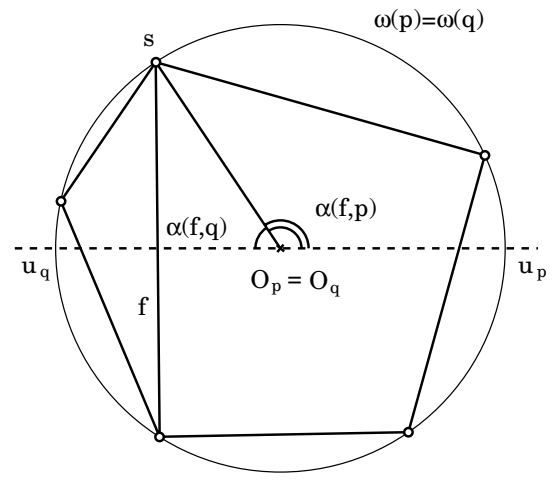

(b)

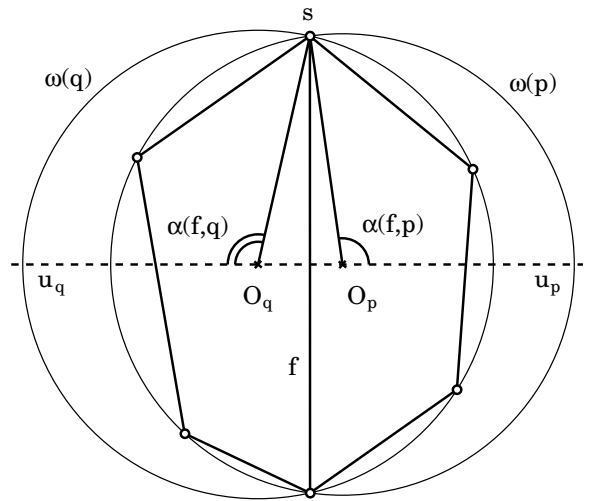

(c)

Fig. 11. The three cases for the proof of Theorem 3 .

Proof. (i) If $f$ is a facet of $\operatorname{Del}(S)$ shared by two bounded regions $p$ and $q$, then from Theorem 1, $f$ is not illegal. Let $O_{p}$ and $O_{q}$ be the respective centers of $\omega(p)$ and $\omega(q)$, and let $O_{p} u_{p}$ and $O_{q} u_{q}$ be the external normals to $f$ with respect to $p$ and $q$, respectively.

Since the region $p$ is not included in the ball $\omega(q)$, the segment of the sphere $\delta(\omega(q))$ that is not on the same side of the hyperplane containing $f$ as $p$, is included in $\omega(p)$ (see Fig. 11(a)). Thus, the center $O_{q}$ of $\omega(q)$ belongs to the half-line $O_{p} u_{p}$, and $O_{p} u_{p} \cap$ $O_{q} u_{q} \neq \emptyset$. Since $\alpha(f, p)$ and $\alpha(f, q)$ are interior angles of the triangle $O_{p} s O_{q}, \beta(f)=$ $\alpha(f, p)+\alpha(f, q)=\pi-\left(s O_{p}, s O_{q}\right)<\pi$.

If $f$ is a facet on the boundary of $\operatorname{conv}(S)$, one of the angles associated to $f$ is zero, and since all associated angles are smaller than $\pi, \beta(f)<\pi$.

(ii) Now let $D$ be a diagram of $\operatorname{Dins}(S)$ which is not Delaunay. From Theorem $1, D$ admits at least one illegal facet $f$ and, with the notations from (i), $p \subset \omega(q)$.

If vertices of $p$ and $q$ are cospherical, $O_{p}=O_{q}$ and $\beta(f)=\alpha(f, p)+\alpha(f, q)=\pi$ (see Fig. 11(b)). 
If the vertices of $p$ and $q$ are not cospherical, then $\alpha(f, p)$ and $\alpha(f, q)$ are the complements of two interior angles of the triangle $O_{p} s O_{q}$ (see Fig. 11(c)). Thus $\beta(f)=$ $\alpha(f, p)+\alpha(f, q)=\pi+\left(s O_{p}, s O_{q}\right)>\pi$.

This property of the Delaunay diagram is in a certain way dual to equiangularity since the Delaunay diagram minimizes the maximum angle coassociated to its facets.

The lifting transformation introduced by Edelsbrunner and Seidel [6], which transforms the $d$-dimensional Delaunay diagram in a $(d+1)$-dimensional convex hull, gives another interpretation of the angle $\beta(f)=\alpha(f, p)+\alpha(f, q)$. The regions $p$ and $q$ are lifted to polytopes lying on two half-hyperplanes which define an angle $\theta(f)$ such that $\theta(f)=\pi$ (resp. $\theta(f)<\pi$ ) if and only if $\beta(f)=\pi$ (resp. $\beta(f)<\pi$ ) (private communication from J.-D. Boissonnat).

\section{Optimality for Self-Centered Diagrams}

A bounded region of a diagram $D$ of $\operatorname{Dins}(S)$ is said to be self-centered if it contains its circumcenter, and the diagram $D$ is said to be self-centered if all its bounded regions are self-centered.

Rajan [11], [12] has proved that every triangulation of a point set in any dimension having each of its triangles self-centered is a Delaunay triangulation. We generalize this result to inscribable diagrams by using coassociated angles.

Theorem 4. Every self-centered diagram is a Delaunay diagram, and is the only one whose angles associated to the facets are all strictly smaller than $\pi / 2$.

Proof. Let $S$ be a $d$-dimensional point set such that $\operatorname{Dins}(S)$ contains a self-centered diagram $D$. Since angles associated to the facets of a self-centered region are all strictly smaller than $\pi / 2$, angles coassociated to the facets of $D$ are strictly smaller than $\pi$, and from Theorem $3 D$ is the Delaunay diagram of $S$. Thus there exists at most one self-centered diagram in $\operatorname{Dins}(S)$, and every non-Delaunay diagram admits at least one non-self-centered region $p$. Therefore, at least one of the angles associated to the facets of $p$ is greater than or equal to $\pi / 2$.

This property of self-centered diagrams is also dual to equiangularity: it minimizes the maximum angle associated to the facets of a diagram. This duality is stronger than the one defined in Section 5 since it is relative to the same angles.

\section{Local Equiangularity in Any Dimension}

Even if equiangularity holds only for planar Delaunay diagrams, there is a property of local equiangularity that characterizes the refinements of the Delaunay diagram in any dimension. We call every inscribable diagram $D$ of $\operatorname{Dins}(S)$ obtained by decomposing some regions and faces of the Delaunay diagram of $S$ a refinement of the Delaunay 


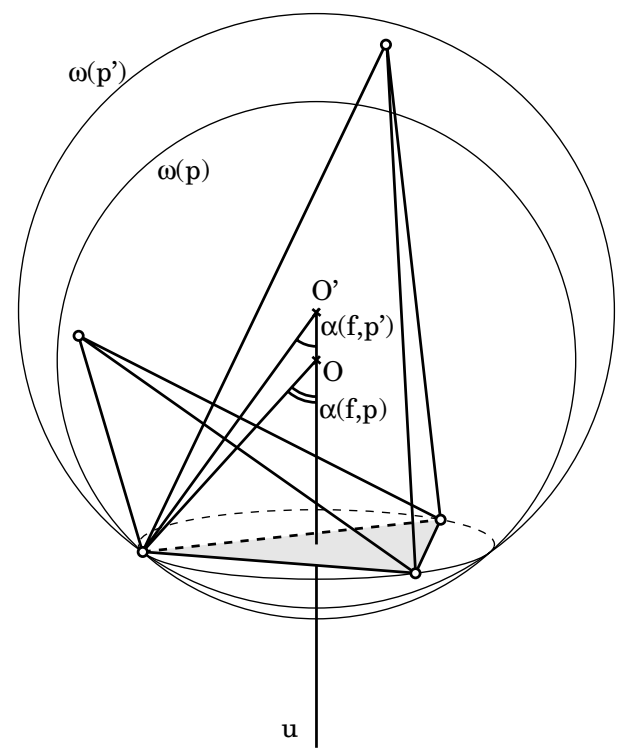

Fig. 12. The grey facet $f$ belongs to both tetrahedrons $p$ and $p^{\prime}$.

diagram of $S$. Therefore every region $p$ of $D$ is such that $\omega(p) \cap S=\emptyset$. In particular, the Delaunay diagram of $S$ and its triangulations are refinements of $\operatorname{Del}(S)$.

Lemma 1. Let $f$ be a facet of a refinement of $\operatorname{Del}(S)$ that belongs to two diagrams $D$ and $D^{\prime}$ of $\operatorname{Dins}(S)$, and let $p$ and $p^{\prime}$ be two bounded regions of $D$ and $D^{\prime}$ respectively that are adjacent to $f$ and on the same side of $f$. If $p$ is a region of a refinement of $\operatorname{Del}(S)$ and if $p^{\prime}$ is not a region of a refinement of $\operatorname{Del}(S)$, then $\alpha\left(f, p^{\prime}\right)<\alpha(f, p)$.

Proof. Let $O$ and $O^{\prime}$ be the respective centers of $\omega(p)$ and $\omega\left(p^{\prime}\right)$, and let $O u$ and $O^{\prime} u$ be the external normals to $f$ with respect to $p$ and $p^{\prime}$ respectively (see Fig. 12). Since $p$ is a region of a refinement of $\operatorname{Del}(S), \omega(p) \cap S=\emptyset$, and since $p^{\prime}$ is not a region of a refinement of $\operatorname{Del}(S)$, the vertices of $p$ that are not vertices of $f$ belong to the open ball $\omega\left(p^{\prime}\right)$. Thus, the center $O$ of $\omega(p)$ belongs to the open half-line $O^{\prime} u$ and $\alpha\left(f, p^{\prime}\right)<\alpha(f, p)$.

Let $\mathcal{A}(f, D)$ be the increasing sequence of the two angles associated to a facet $f$ of a diagram $D$ of $\operatorname{Dins}(S)$. The diagram $D$ is said to be locally equiangular in $f$ if, for every diagram $D^{\prime}$ of $\operatorname{Dins}(S)$ that admits $f$ as a facet, $\mathcal{A}\left(f, D^{\prime}\right) \leq \mathcal{A}(f, D)$. Moreover, $D$ is said to be locally equiangular (with respect to its facets) if it is locally equiangular in all its facets.

Theorem 5. The refinements of the Delaunay diagram are locally equiangular. 
Proof. Let $D$ be a refinement of $\operatorname{Del}(S)$, let $f$ be a facet of $D$, and let $p$ and $q$ be the two regions of $D$ sharing $f$. Let $D^{\prime}$ be a diagram of $\operatorname{Dins}(S)$ that admits $f$ as a facet and let $p^{\prime}$ (resp. $q^{\prime}$ ) be the region of $D^{\prime}$ adjacent to $f$ and on the same side of $f$ as $p$ (resp. $q$ ).

If $p^{\prime}$ is the unbounded region of $D^{\prime}, p$ is also the unbounded region of $D$ and $\alpha\left(f, p^{\prime}\right)=$ $\alpha(f, p)=0$. If $p^{\prime}$ is a bounded region of a refinement of $\operatorname{Del}(S)$, then $\omega\left(p^{\prime}\right) \cap S$ is empty and, since $\omega(p) \cap S$ is also empty, vertices of $p$ and $p^{\prime}$ are cospherical. Thus the centers $O$ and $O^{\prime}$ of $\omega(p)$ and $\omega\left(p^{\prime}\right)$ coincide and $\alpha\left(f, p^{\prime}\right)=\alpha(f, p)$. If $p^{\prime}$ is not a region of a refinement of $\operatorname{Del}(S)$, then from Lemma $1 \alpha\left(f, p^{\prime}\right)<\alpha(f, p)$.

It results in all cases that $\alpha\left(f, p^{\prime}\right) \leq \alpha(f, p)$. In the same way we show that $\alpha\left(f, q^{\prime}\right) \leq$ $\alpha(f, q)$ and thus $\mathcal{A}\left(f, D^{\prime}\right) \leq \mathcal{A}(f, D)$.

Conjecture 1. The refinements of the Delaunay diagram are the only inscribable diagrams that are locally equiangular.

Prooffor Some Particular Cases. (i) A diagram of $\operatorname{Dins}(S)$ that is locally equiangular and that admits at least one facet of a refinement of $\operatorname{Del}(S)$ is a refinement of $\operatorname{Del}(S)$. Indeed, let $D$ be a diagram of $\operatorname{Dins}(S)$ that is not a refinement of $\operatorname{Del}(S)$ but that admits at least one facet of a refinement of $\operatorname{Del}(S)$. If the regions adjacent to this facet are regions of a refinement of $\operatorname{Del}(S)$, all facets of these regions are also facets of a refinement of $\operatorname{Del}(S)$. Since $D$ is not a refinement of $\operatorname{Del}(S), D$ admits at least one facet $f$ of a refinement of $\operatorname{Del}(S)$ adjacent to a region that is not a region of a refinement of $\operatorname{Del}(S)$; from Lemma 1 such a diagram is not locally equiangular in $f$.

(ii) A planar inscribable diagram that is locally equiangular is a refinement of Delaunay. Indeed, in whichever way you decompose the convex hull of a planar point set in inscribable regions, edges adjacent to the unbounded region are the same and thus are Delaunay edges.

(iii) More generally in $d$-dimensional space, if $\delta(\operatorname{conv}(S))$ admits at least one simplicial facet, then this facet belongs to every diagram of $\operatorname{Dins}(S)$, and the refinements of $\operatorname{Del}(S)$ are the only locally equiangular diagrams of $\operatorname{Dins}(S)$.

(iv) However, there exist inscribable diagrams that admit no facets of a refinement of Delaunay, as shown in the following example. Let $S$ be a set of eight sites in threedimensional space that generate a convex polyhedron whose facets are quadrangles (see Fig. 13). The facet 1234 is a diamond whose diagonal 13 is strictly greater than 24 , and edges 15, 26, 48, and 37 are orthogonal to 1234 and such that $|15|<|26|=|48|<|37|$.

Figure 13(b) shows the diagram $D$ of $\operatorname{Dins}(S)$ that contains no Delaunay facet. However, in this example it is easy to show that $D$ is not equiangular and that $\operatorname{Del}(S)$ is the only equiangular diagram of $\operatorname{Dins}(S)$.

\section{Coassociated Solid Angles in Dimension $d \geq 3$}

In the previous sections we have studied the properties of Delaunay diagrams by using line angles. In order to establish the same properties with solid angles, we have to associate a solid angle $\alpha_{\mathrm{s}}(f, p)$ to every facet $f$ of an inscribable diagram $D$ with respect to each region $p$ adjacent to $f$. However, we cannot choose for $\alpha_{\mathrm{s}}(f, p)$ the solid angle under 


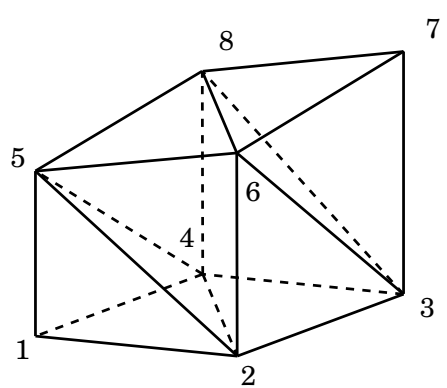

(a)

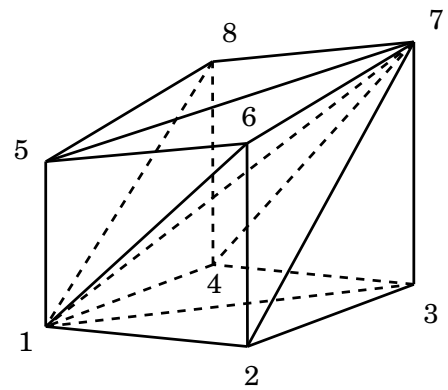

(b)

Fig. 13. The Delaunay diagram (a) and a diagram without any Delaunay facet (b) of a three-dimensional point set.

which the facet $f$ is seen from the circumcenter $O$ of $p$. Indeed, if $f$ is not self-centered, this angle does not vary monotonously when $O$ passes through the hyperplane containing $f$ (see, for example, facet $s_{1} s_{2} s_{3}$ in Fig. 15).

Let $c_{1}, c_{2}, \ldots, c_{m}$ be the facets of $f$, that is, the $(d-2)$-faces of $f$, and $\forall i \in\{1, \ldots, m\}$, let $s_{i}^{\prime}$ be the intersection of the external normal to $c_{i}$ with respect to $f$ with the sphere $\delta(\omega(p))$ (see Fig. 14).

If $O$ is the center of $\omega(p)$ and $O u$ is the external normal to $f$ with respect to $p$, then the solid angle $\alpha_{\mathrm{s}}(f, p)$ at vertex $O$ of the polytope $O s_{1}^{\prime} s_{2}^{\prime} \cdots s_{m}^{\prime}$ that cuts the half-line $O u$ is said to be associated to the facet $f$ with respect to the region $p$. We pose $\alpha_{\mathrm{s}}(f, p)=0$ if $p$ is unbounded.

Lemma 2. If $h$ is the circumcenter of $f$, then the solid angle $\alpha_{\mathrm{s}}(f, p)$ is a continuous, increasing function of the algebraic measure $\overline{h O}$ on the oriented straight line $h u$.
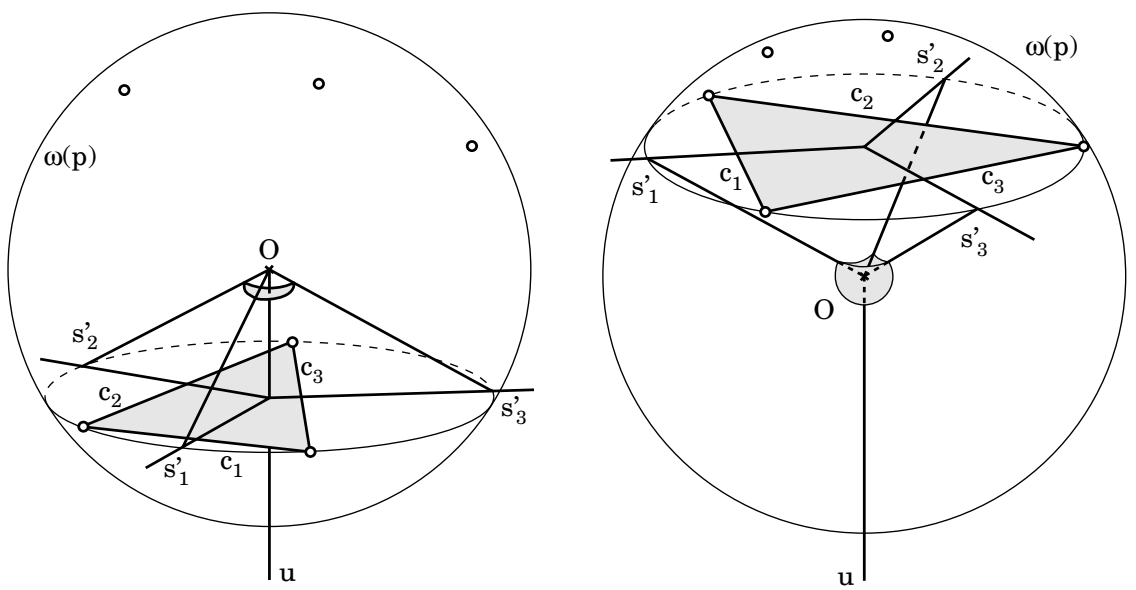

Fig. 14. In both figures the facet $f$ is in grey and the region $p$ is "above" this facet. $p$ is only represented by its vertices and its circumball $\omega(p)$. 

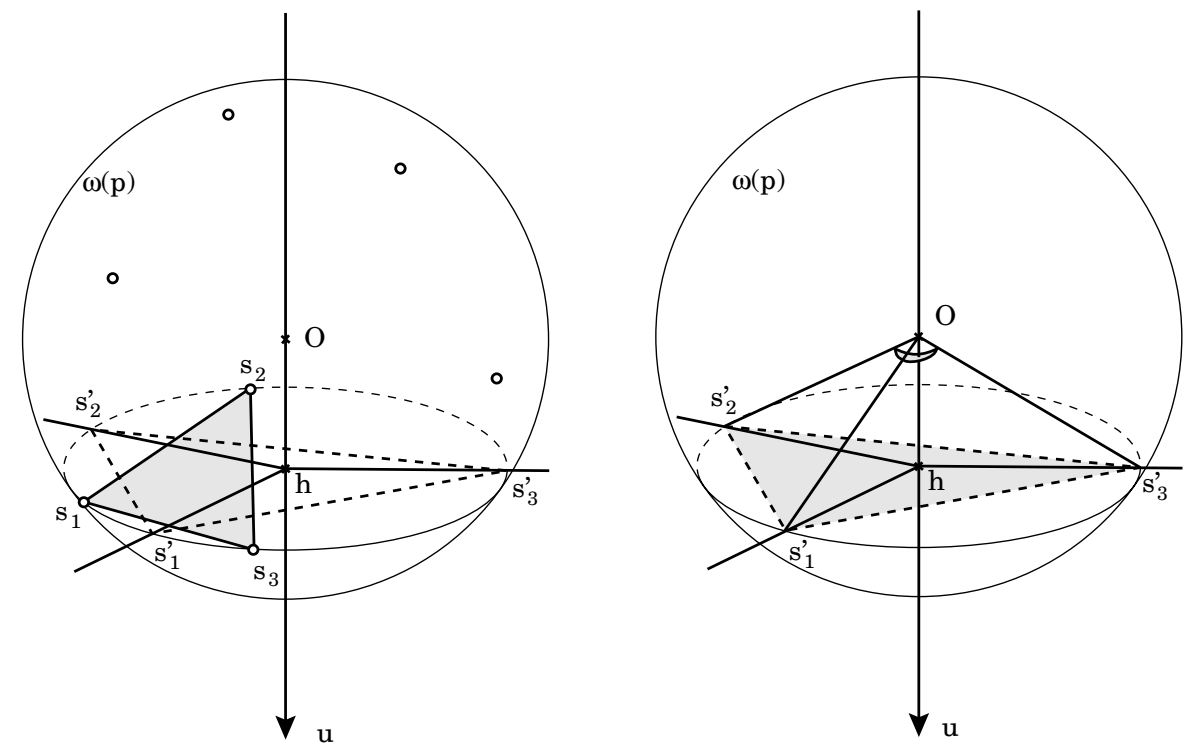

Fig. 15. The facet $s_{1}^{\prime} s_{2}^{\prime} s_{3}^{\prime}$ is self-centered, whereas $s_{1} s_{2} s_{3}$ is not self-centered.

Proof. It is straightforward to show that the facet $f^{\prime}$ with vertices $s_{1}^{\prime}, \ldots, s_{m}^{\prime}$ is selfcentred, that is, the center $h$ of $\omega\left(f^{\prime}\right)=\omega(f)$ belongs to $f^{\prime}$ (see Fig. 15). It follows that the solid angle $\alpha_{\mathrm{s}}(f, p)$ is equal to $\gamma / 2$ when $\overline{h O}$ is equal to zero (where $\gamma$ is the solid angle that sees the whole $d$-dimensional space). Moreover, $\alpha_{\mathrm{s}}(f, p)$ decreases and tends toward zero when $\overline{h O}$ tend toward $-\infty$, and $\alpha_{\mathrm{s}}(f, p)$ increases and tends toward $\gamma$ when $\overline{h O}$ tends toward $+\infty$. Hence $\alpha_{\mathrm{s}}(f, p)$ is a continuous increasing function of $\overline{h O}$.

The example of Section 4 shows again that equiangularity of Delaunay diagrams in higher dimensions cannot be established with these angles. The property of coassociated angles can however be generalized as follows. If $p$ and $q$ are the two regions sharing the facet $f$, let $\beta_{\mathrm{s}}(f)=\alpha_{\mathrm{s}}(f, p)+\alpha_{\mathrm{s}}(f, q)$ be the solid angle coassociated to $f$.

Theorem 6. In any dimension $d, \operatorname{Del}(S)$ is the only diagram of $\operatorname{Dins}(S)$ whose solid angles coassociated to the facets are all strictly smaller than the solid angle $\gamma$ that sees the whole d-dimensional space.

Proof. (i) If $f$ is a facet adjacent to two bounded regions $p$ and $q$ in $\operatorname{Del}(S)$, then from Theorem $1 f$ is not illegal. Let $O_{p}$ and $O_{q}$ be the respective centers of $\omega(p)$ and $\omega(q)$, and let $O_{p} u_{p}$ and $O_{q} u_{q}$ be the external normals to $f$ with respect to $p$ and $q$ respectively. Since the region $p$ is not included in $\omega(q), O_{q}$ belongs to the half-straight line $O_{p} u_{p}$ (see Fig. 16(a)). From Lemma 2, the angle $\alpha_{\mathrm{s}}(f, p)$ is smaller than the supplement of $\alpha_{\mathrm{s}}(f, q)$ and $\beta_{\mathrm{s}}(f)=\alpha_{\mathrm{s}}(f, p)+\alpha_{\mathrm{s}}(f, q)<\gamma$. 


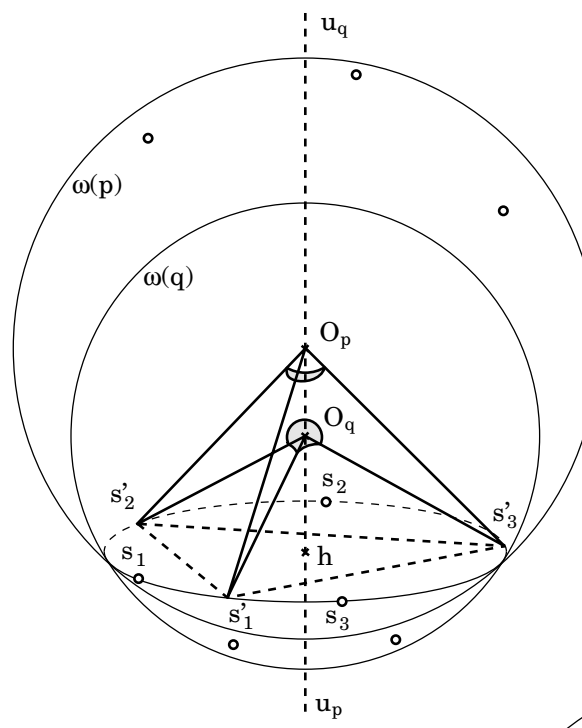

(a)

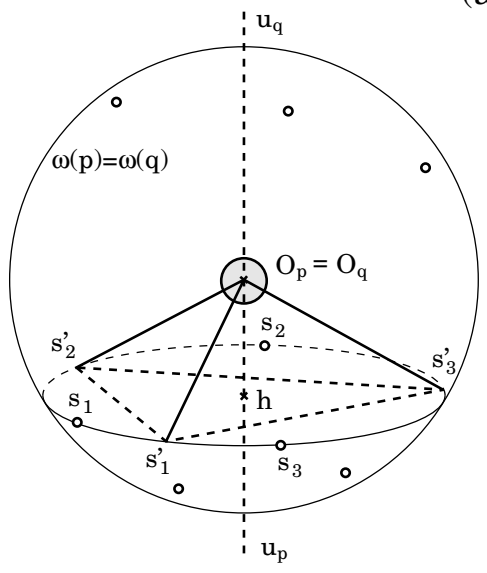

(b)

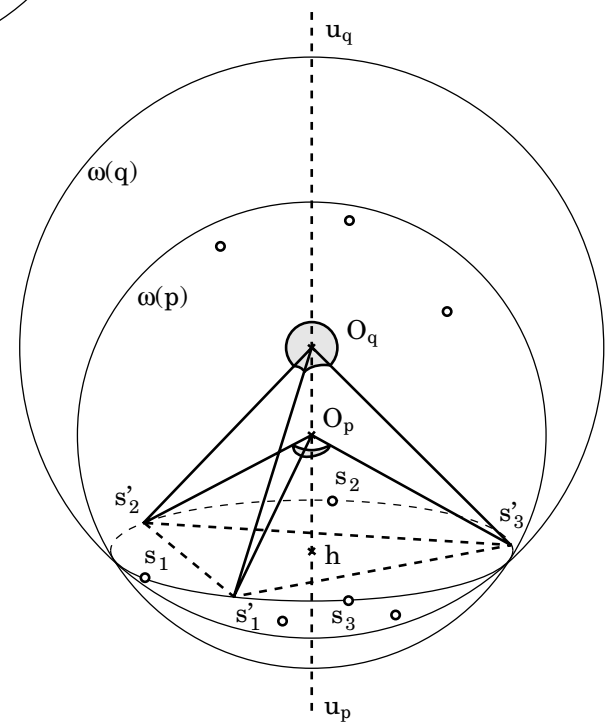

(c)

Fig. 16. The region $p$ is "above" the facet $f$ with vertices $s_{1}, s_{2}, s_{3}$ and $q$ is "below" this facet.

If $f$ is on the boundary of the convex hull of $S$, one of the angles associated to $f$ is zero, and since the solid angles associated to the facets are all smaller than $\gamma, \beta_{\mathrm{s}}(f)$ $<\gamma$.

(ii) Now let $D$ be a non-Delaunay diagram. From Theorem $1 D$ admits at least one illegal facet $f$ and, with the notations of (i), $p \subset \omega(q)$. If the vertices of $p$ and $q$ are cospherical, then $O_{p}=O_{q}, \alpha_{\mathrm{s}}(f, p)$ is the supplement of $\alpha_{\mathrm{s}}(f, q)$, and $\beta_{\mathrm{s}}(f)=\gamma$ (see Fig. 16(b)). If the vertices of $p$ and $q$ are not cospherical, then the angle $\alpha_{\mathrm{s}}(f, p)$ is greater than the supplement of $\alpha_{\mathrm{s}}(f, q)$ and $\beta_{\mathrm{s}}(f)>\gamma$ (see Fig. 16(c)). 
It is straightforward to show that results given in Sections 6 and 7 also hold with these solid angles.

\section{Angular Properties of Farthest Point Delaunay Diagrams}

We now define the set of diagrams that enables us to characterize the farthest point Delaunay diagram $\operatorname{FDD}(S)$ of a $d$-dimensional point set $S$.

Let CH_Dins $(S)$ be the set of inscribable diagrams whose vertices are the vertices of the convex hull of $S$. A facet $f$ of such a diagram is said to be legal if the two regions $p$ and $q$ having $f$ as a common facet are bounded and if vertices of $p \backslash f$ do not belong to the open ball $\omega(q)$. If no more than $d+1$ sites are cospherical, legality is equivalent to nonillegality.

Theorem 7. In a d-dimensional euclidean space $E, \operatorname{FDD}(S)$ is the only diagram of CH_Dins $(S)$ without legal facets.

Proof. (i) $\operatorname{FDD}(S)$ is a partition of $E$ whose every bounded region $p$ is inscribable, convex, and such that $S \cap \omega(p)=S \backslash \delta(p)$. Thus, $\operatorname{FDD}(S)$ has no legal facet.

(ii) If $D$ is a diagram of CH_Dins $(S)$ distinct from $\operatorname{FDD}(S)$, there exist a region $p$ of $D$ and a site $s$ of $S \backslash \delta(p)$ such that $s \notin \omega(p)$. Let $z$ be a point of $p$ such that the open straight-line segment $s z$ does not cut any $h$-face of $D$ with dimension lower than $d-1$ (see Fig. 17 for $d=2$ ). Such a point $z$ always exists since the number of $h$-faces is finite. We now show that $D$ admits at least one legal facet, by induction on the number of facets that are cut by $s z$.

Since $s \notin \bar{p}$ and $z \in p$, there exists at least one facet $f$ of $p$ cut by $s z$. If $s z$ cuts exactly one facet $f$ of $D$, then $f$ is a facet of $p, s$ is a vertex of the region $q$ sharing facet $f$ with $p$, and $f$ is legal.

We take the following induction hypothesis: if $s z$ cuts $k$ facets, then $s z$ cuts at least one legal facet. Suppose now that $s z$ cuts $k+1$ facets, and let $f$ be the facet of $p$ cut

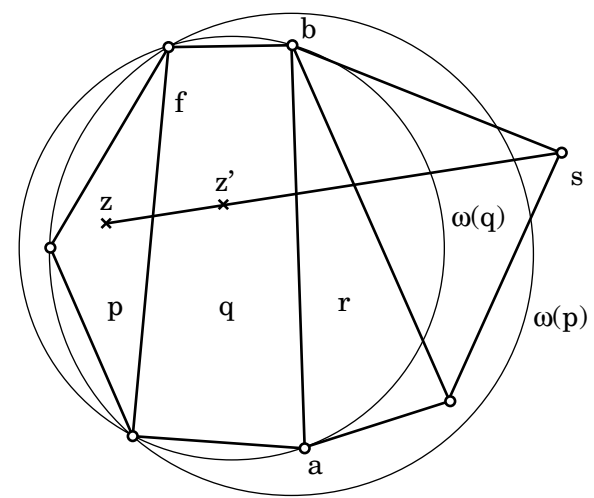

Fig. 17. Since $\omega(q)$ does not contain any vertex of $r$, the edge $a b$ is legal. 
by $s z$ and let $q$ be the region of $D$ sharing facet $f$ with $p$. If the vertices of $q \backslash f$ do not belong to $\omega(p)$, then the facet $f$ is legal and the result is directly proved. Otherwise, since $s \notin \omega(p)$ and since $s$ and $z$ are on both sides of $f, s \notin \omega(q)$. If $z^{\prime}$ is a point of $q \cap s z, s z^{\prime}$ cuts $k$ facets of $D$ but does not cut any $h$-face of $D$ with dimension lower than $d-1$. From the induction hypothesis, $s z^{\prime}$ cuts at least one legal facet and $s z$ also cuts this facet.

If $D$ is a diagram of $C H \_\operatorname{Dins}(S), p$ is a bounded region of $D$, and $f$ is a facet of $p$, then we associate to $f$ with respect to $p$ the same angle $\alpha^{\prime}(f, p)=\alpha(f, p)$ as in Section 4 . In the particular case where the region $p$ is unbounded, since the hyperplane of $f$ is the limit of a sphere that contains $S$, we pose $\alpha^{\prime}(f, p)=\pi$. Let $\mathcal{A}^{\prime}(D)$ be the increasing sequence of the angles smaller than $\pi$ associated to the facets of $D$.

In order to establish a property of anti-equiangularity of the farthest point Delaunay diagram, we only consider the subset of diagrams of $\mathrm{CH}_{-} \operatorname{Dins}(S)$ such that the circumsphere of every region passes only through sites that are vertices of this region. A diagram $D$ of this subset is said to be anti-equiangular if, for every diagram $D^{\prime}$ of the subset, $\mathcal{A}^{\prime}(D) \leq \mathcal{A}^{\prime}\left(D^{\prime}\right)$.

Theorem 8. For a planar point set $S, \operatorname{FDD}(S)$ is the only anti-equiangular diagram of the reduced $\mathrm{CH}$ _Dins $(S)$.

Proof. Using the same arguments as in the proof of Theorem 2, we show that every diagram of the reduced $\mathrm{CH} \operatorname{Dins}(S)$ that admits at least one legal facet is not antiequiangular. From Theorem 7, it results that $\operatorname{FDD}(S)$ is the only anti-equiangular diagram of the reduced CH_Dins $(S)$.

The anti-equiangular diagrams of the whole $\mathrm{CH} \_\operatorname{Dins}(S)$ are farthest point Delaunay triangulations. Indeed, angles associated to the facets of the farthest point Delaunay diagram form a subset of those associated to the facets of a farthest point Delaunay triangulation.

As in Section 5, let $\beta^{\prime}(f)$ be the angle coassociated to a facet $f$ of a diagram of CH_Dins $(S)$.

Theorem 9. For ad-dimensional point set $S, \operatorname{FDD}(S)$ is the onlydiagram of CH_Dins $(S)$ whose coassociated angles are all strictly greater than $\pi$.

Proof. From the proof of Theorem 3, the angle $\beta^{\prime}(f)$ coassociated to any facet $f$ that is not on the boundary of $\operatorname{conv}(S)$ is either smaller than or equal to $\pi$ if $f$ is legal, or strictly greater than $\pi$ otherwise. Moreover, if $f$ is on $\delta(\operatorname{conv}(S))$, then one of its associated angles is equal to $\pi$ and $\beta^{\prime}(f)>\pi$.

From Theorem 7 it follows that all angles coassociated to facets of $\operatorname{FDD}(S)$ are strictly greater than $\pi$, and that $\operatorname{FDD}(S)$ is the only diagram of CH_Dins $(S)$ with this property.

The interested reader can show that the notions of coassociated solid angles, of local 
equiangularity, and of self-centered diagrams can also be modified to characterize farthest point Delaunay diagrams in any dimension.

\section{Conclusion}

In order to characterize the regularity of the Delaunay diagram in any dimension, we have compared this diagram with all those whose regions are convex and inscribable polytopes. By associating two angles to each edge of a planar inscribable diagram, we have generalized the equiangularity property to the Delaunay diagram and have shown that it is the only equiangular diagram.

However, natural generalizations of these angles do not allow us to establish equiangularity of the Delaunay diagram in dimension greater than two. Nevertheless, we have presented other angular optimality results for the Delaunay diagram in any dimension, both with line angles and with solid angles.

In the last section we have given angular properties of the farthest point Delaunay diagram. The question that arises is to know if such properties can also be extended to any order- $k$ Delaunay diagram of a set of $n$ sites. In [13] we have shown that, for $2 \leq k \leq n-2$, these diagrams contain both Delaunay and farthest point Delaunay subdiagrams. Since these two kinds of diagrams have opposite angular properties, generalization to any order $k$ is not possible.

We hope that our results will help to determine a Delaunay triangulation from any triangulation by local flips, a problem which remains open in dimension greater than two.

\section{Acknowledgments}

The authors wish to thank the referees and Stéphane Rivière whose advice has greatly helped to enhance the presentation of this paper.

\section{References}

1. F. Aurenhammer. A new duality result concerning Voronoi diagrams. Discrete Comput. Geom., 5:243-254, 1990 .

2. F. Aurenhammer. Voronoi diagrams: a survey of a fundamental geometric data structure. ACM Comput. Surv., 23:345-405, 1991.

3. F. Aurenhammer and O. Schwarzkopf. A simple on-line randomized incremental algorithm for computing higher-order Voronoi diagrams. Internat. J. Comput. Geom. Appl., 2:363-381, 1992.

4. B. Delaunay. Sur la sphère vide. à la mémoire de Georges Voronoï. Bull. Acad. Sci. USSR: Cl. Sci. Math. Natur., 7:793-800, 1934.

5. H. Edelsbrunner. Algorithms in Combinatorial Geometry, volume 10 of EATCS Monographs on Theoretical Computer Science. Springer-Verlag, Heidelberg, 1987.

6. H. Edelsbrunner and R. Seidel. Voronoi diagrams and arrangements. Discrete Comput. Geom., 1:25-44, 1986.

7. D. Eppstein. The farthest point Delaunay triangulation minimizes angles. Comput. Geom. Theory Appl., 1:143-148, 1992. 
8. C. L. Lawson. Software for $C^{1}$ surface interpolation. In J. R. Rice, editor, Mathematics Software III, pages 161-194. Academic Press, New York, 1977.

9. A. Okabe, B. Boots, and K. Sugihara. Spatial Tesselations: Concepts and Applications of Voronoi Diagrams. Wiley, Chichester, 1992.

10. F. P. Preparata and M. I. Shamos. Computational Geometry: An Introduction. Springer-Verlag, New York, 1985.

11. V. T. Rajan. Optimality of the Delaunay triangulation in $R^{d}$. In Proc. 7th Ann. ACM Symp. Comput. Geom., pages 357-363, 1991.

12. V. T. Rajan. Optimality of the Delaunay triangulation in $R^{d}$. Discrete Comput. Geom., 12:189-202, 1994.

13. D. Schmitt. Sur les diagrammes de Delaunay et de Voronoï d'ordre $k$ dans le plan et dans l'espace. Ph.D. thesis, Université de Haute-Alsace, Mulhouse, 1995.

14. D. Schmitt and J.-C. Spehner. On Delaunay and Voronoi diagrams of order $k$ in the plane. In Proc. $3 r d$ Canad. Conf. Comput. Geom., pages 29-32, 1991.

15. D. Schmitt and J.-C. Spehner. On equiangularity of Delaunary diagrams in every dimension. In Proc. 5th Canad. Conf. Comput. Geom., pages 346-351, 1993.

Received April 25, 1996, and in revised form July 31, 1997, and March 18, 1998. 